\title{
Manejo y desenlaces de la pancreatitis aguda en un hospital de cuarto nivel (Huila, Colombia), 3 años de experiencia
}

\section{Outcomes of Three Years of Experience Managing Acute Pancreatitis at a Fourth Level Hospital in Huila, Colombia}

\author{
Leidy Juliana Puerto H., ${ }^{\text {* }}$ Rolando Medina R., ${ }^{1}$ Luis Ramiro Núñez R., ${ }^{1}$ Héctor Conrado Jiménez S., ${ }^{1}$ Justo Germán Olaya R., \\ Juan Felipe Sanjuán M., ${ }^{1}$ Sergio Andrés Puerto H., ${ }^{2}$ Carlos Mauricio Martínez M. ${ }^{3}$ María Mónica Medina C. ${ }^{4}$
}

\footnotetext{
Hospital Universitario Hernando Moncaleano Perdomo de Neiva. Neiva, Colombia.

2 Hospital San José. Bogotá D. C., Colombia.

3 Hospital San Juan de Dios. Ituango, Antioquia Colombia.

${ }^{4}$ Hospital El Tunal. Bogotá D. C., Colombia.

*Correspondencia: puertojuliana@hotmail.com.
}

Fecha recibido: $\quad 06 / 04 / 18$ Fecha aceptado: 15/06/18

\begin{abstract}
Resumen
Objetivo: describir el comportamiento clínico y quirúrgico de los pacientes con pancreatitis aguda manejados en un centro hospitalario de cuarto nivel. Método: se realizó un estudio descriptivo retrospectivo analítico de los pacientes con diagnóstico de pancreatitis aguda que ingresaron al Hospital Universitario de Neiva en el período comprendido entre el 1 de enero del 2015 al 31 de julio del 2017. Resultados: se identificaron 138 pacientes que ingresaron con impresión diagnóstica de pancreatitis aguda, de los cuales se confirmó el diagnóstico en 130 pacientes y se excluyeron 21 pacientes por no cumplir con los criterios de inclusión. Según la clasificación de Atlanta 2012, 73 (67 \%) pacientes cursaban con una pancreatitis leve, 17 (16\%) con pancreatitis moderadamente severa y $19(17 \%)$ con pancreatitis severa. La principal causa de pancreatitis encontrada fue la biliar. La prueba de chi cuadrado $\left(X^{2}\right)$ mostró una significancia estadística para la edad como factor de riesgo para pancreatitis severa $(p=0,04)$. De la totalidad de pacientes, en 8 pacientes se documentó necrosis pancreática, de los cuales 5 fueron manejados quirúrgicamente, con un abordaje por laparotomía en 3 pacientes y 2 por laparoscopia, con una mortalidad del $37,5 \%$ en los pacientes con necrosis pancreática manejados quirúrgicamente. De los 109 pacientes incluidos en el estudio, 100 pacientes $(91,74 \%)$ resolvieron su cuadro de pancreatitis y egresaron vivos de la institución, para una mortalidad reportada en 9 pacientes $(8,25 \%)$, quienes cursaron con pancreatitis aguda severa. Conclusión: el estudio presentado es de tipo descriptivo estableciendo las características demográficas y el curso de la enfermedad de los pacientes con pancreatitis aguda incluidos las complicaciones y el manejo de estas. Con este estudio no se pretende estandarizar causalidad ni manejo de la patología descrita, solo se describe la experiencia realizada en un hospital de cuarto nivel tratando de contribuir con el desarrollo de estudios de cohortes y ensayos clínicos que permitan establecer guías de manejo con el fin de mejorar los desenlaces de los pacientes con pancreatitis aguda.
\end{abstract}

Palabras clave

Pancreatitis aguda, necrosis pancreática, pancreatitis aguda severa.

\section{INTRODUCCIÓN}

La pancreatitis aguda es una enfermedad inflamatoria del páncreas que causa un gran número de ingresos a los servicios de urgencias alrededor del mundo, se caracteriza por presentar un amplio espectro de manifestaciones clínicas, que van desde síntomas leves autolimitados hasta procesos con insuficiencia orgánica múltiple y choque (1). Su inci- dencia anual está entre 13 a 45 individuos por cada 100000 habitantes (2), y las principales causas de pancreatitis aguda se asocian con la patología biliar y consumo de alcohol, seguida por otras comorbilidades como dislipidemias, algunos fármacos y procesos infecciosos.

A pesar de las mejoras en el acceso a la atención, las imágenes y las técnicas de intervención, la pancreatitis aguda sigue estando asociada con una morbimortalidad significativa que 


\begin{abstract}
Objective: Our objective was to describe the clinical and surgical behavior of patients with acute pancreatitis managed at a fourth level hospital center. Method: This is a descriptive, retrospective and analytical study of patients diagnosed with acute pancreatitis who entered the University Hospital of Neiva in the period from January 1, 2015 to July 31, 2017. Results: We identified 138 patients who had been admitted with a diagnosis of acute pancreatitis. Of these, the diagnosis was confirmed for 130 patients, but 21 patients were excluded because they did not meet the inclusion criteria. According to the 2012 Atlanta classification, 73 (67\%) patients had mild pancreatitis, $17(16 \%)$ had moderately severe pancreatitis, and $19(17 \%)$ had severe pancreatitis. The main cause of pancreatitis was biliary. The chi-square test showed statistical significance for age as a risk factor for severe pancreatitis $(p=0.04)$. Pancreatic necrosis was documented in 8 patients, five of whom were managed surgically. This included three laparotomies and two laparoscopic operations. The mortality rate for patients with surgically managed pancreatic necrosis was $37.5 \%$. Of the 109 patients included in the study, the resolved their pancreatitis of 100 patients (91.74\%) was resolved and they left the institution alive. Nine patients (8.25\%), all of whom had severe acute pancreatitis, died. Conclusion: This descriptive study established the demographic characteristics and clinical course of patients with acute pancreatitis including complications and management of complications. This study is not intended to standardize causality or management of acute pancreatitis, but rather only describes the experience of a fourth level hospital in order to contribute to the development of cohort studies and clinical trials that will allow establishment of management guidelines to improve the outcomes of patients with acute pancreatitis.
\end{abstract}

\title{
Keywords
}

Acute pancreatitis, pancreatic necrosis, severe acute pancreatitis.

depende de las comorbilidades del paciente y la severidad del cuadro clínico, llegando a estar entre el $7 \%$ y $40 \%$ en los peores casos (3). Por este motivo, se han realizado múltiples esfuerzos para caracterizar y definir epidemiológicamente diferentes guías y recomendaciones sobre el manejo de estos pacientes, esto se refleja en la aparición de aproximadamente 14 guías de manejo entre los años 2004 y 2014 (4); a pesar de esto, es frecuente encontrar en la práctica médica a nivel mundial criterios no tan claros en cuanto al manejo y los procedimientos indicados en cada uno de los grados de esta patología; e igualmente, la no adherencia en la práctica clínica de dichas recomendaciones.

Lo anterior demuestra la importancia de continuar realizando diversos estudios en cuanto al comportamiento de esta enfermedad y los resultados obtenidos por los diferentes grupos tratantes, trabajos que permitan consolidar recomendaciones claras y aplicables a los diferentes niveles de atención hospitalaria y, de este modo, mejorar los resultados y el pronóstico de la población afectada.

En el presente trabajo se presentan las características sociodemográficas y clínicas de los pacientes atendidos en un hospital de III nivel de la ciudad de Neiva, con diagnóstico de pancreatitis leve, moderadamente severa y severa; se describe su comportamiento clínico y sus resultados posteriores al manejo instaurado en esta institución.

\section{MATERIALES Y MÉTODO}

Se realizó un estudio descriptivo retrospectivo analítico de los pacientes con diagnóstico de pancreatitis aguda que ingresaron al Hospital Universitario de Neiva en el período comprendido entre el 1 de enero del 2015 al 31 de julio del 2017, los datos se recogieron de los registros médicos del Hospital Universitario de Neiva (HUN). Los datos se organizaron en tablas, llevando a cabo una descripción de la población estudiada, la clasificación, las complicaciones, la morbilidad y mortalidad, así como su relación con las diferentes variables clínicas de los pacientes.

\section{RESULTADOS}

Se encontraron 138 pacientes que ingresaron al hospital universitario de Neiva con impresión diagnóstica de pancreatitis aguda y se confirmó el diagnóstico en 130 pacientes, de los cuales se excluyeron 21 pacientes por no cumplir con los criterios de inclusión (Figura 1).

El total de pacientes estudiados fue de 109, de los cuales 43 correspondieron al sexo masculino y 68 al sexo femenino. La edad de los pacientes osciló en un rango entre 13 a 94 años, para un total de 8 pacientes menores de 25 años, 58 pacientes entre 25 y 59 años y 43 mayores de 60 años. El promedio de índice de masa corporal (IMC) de estos pacientes fue de $26,7 \mathrm{~kg} / \mathrm{m}^{2}$, cursando con sobrepeso 43 pacientes $(39,8 \%)$ y obesidad 24 pacientes $(22 \%)$, el resto de pacientes estuvo en el rango de peso normal (Tabla 1 ).

Dentro de las comorbilidades presentadas, la más frecuente fue la HTA en 28 pacientes $(25,6 \%)$, seguida por la patología biliar en 11 pacientes $(10,09 \%)$, diabetes mellitus (DM) en 8 pacientes $(7,33 \%)$, cardiopatía en 5 pacientes $(4,58 \%)$, embarazo en 3 pacientes $(2,75 \%)$ y 42 pacientes 


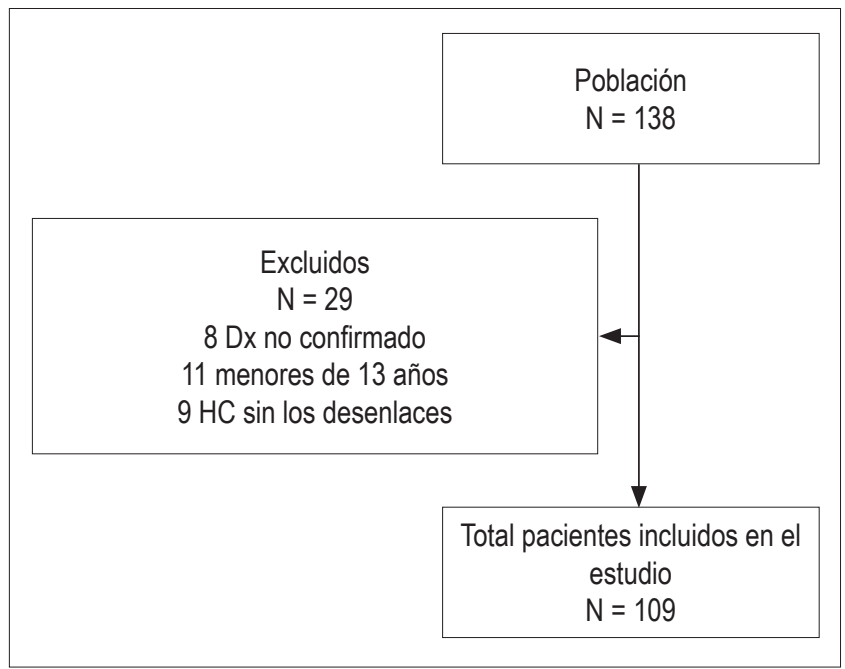

Figura 1. Metodología de selección de pacientes. Dx: diagnóstico; HC: historia clínica.

no presentaron ninguna comorbilidad. Se encontró amilasa positiva en 93 pacientes (85,3\%), negativa en 11 pacientes $(10,09 \%)$ y sin datos en 5 pacientes $(4,58 \%)$, con un promedio de valor de amilasa de 1900 (Tabla 1).

Dentro de las escalas de severidad descritas, se calculó el Ranson al ingreso con un valor promedio de 1,14 , encontrando ausencia de datos en 20 pacientes (18,3\%); se calculó $<3$ en 81 pacientes $(74,3 \%)$ y $>3$ en 8 pacientes $(7,3 \%)$. El Ranson a las 48 horas fue difícil de calcular dada la ausencia de datos en la historia clínica, encontrando Ranson $<3$ en 27 pacientes $(24,7 \%),>3$ en 10 pacientes $(9,1 \%)$, sin datos para calcular 72 pacientes $(66 \%)$ y un valor promedio de 1,54. Según la clasificación de Marshall calculada se encontraron 53 pacientes $(48,6 \%), \geq 2$ en 14 pacientes $(12,8 \%)$ y sin datos para calcular en 52 pacientes $(47,7 \%)$. El APACHE II al ingreso reportado fue $<8$ en 54 pacientes $(49,5 \%)$, $>8$ en 41 pacientes $(37,6 \%)$ y sin datos en 14 pacientes $(13,8 \%)$, con un promedio de APACHE II al ingreso en los 109 pacientes incluidos en el estudio de 7,49. El APACHE II a las 48 horas se encontró $<8$ en 13 pacientes $(11,9 \%)$, $>8$ en 13 pacientes $(11,9 \%)$ y sin datos para calcularlo en 83 pacientes $(76,1 \%)$, con un valor promedio de 9,76 (Tabla 1).

Según la clasificación de Atlanta 2012, de la totalidad de pacientes, 73 (67\%) cursaban con una pancreatitis leve, 17 (16\%) con pancreatitis moderada y 19 (17\%) con pancreatitis severa (Tabla 1).

La principal causa de pancreatitis encontrada fue la biliar en 91 pacientes (83,2 \%), 4 pacientes presentaron pancreatitis posterior a la realización de colangiopancreatografía retrógrada endoscópica (CPRE), y de los pacientes
Tabla 1. Características generales de los pacientes

\begin{tabular}{|c|c|c|c|c|}
\hline Característica & Total (\%) & Promedio & Rango & Moda \\
\hline $\begin{array}{l}\text { Edad } \\
\qquad \begin{array}{l}<5 \text { años } \\
25-59 \text { años } \\
\geq 60\end{array}\end{array}$ & $\begin{array}{c}8(7,34) \\
58(53,2) \\
43(39,4)\end{array}$ & 50,7 & $13-94$ & 62 \\
\hline $\begin{array}{l}\text { Sexo } \\
\text { Femenino } \\
\text { Masculino }\end{array}$ & $\begin{array}{c}68(62) \\
41(37,6)\end{array}$ & & & \\
\hline $\begin{array}{l}\text { Comorbilidades } \\
\text { HTA } \\
\text { DM2 } \\
\text { Cardiopatía } \\
\text { Embarazo } \\
\text { Patología biliar }\end{array}$ & $\begin{array}{c}28(25,6) \\
8(7,3) \\
5(4,5) \\
3(2,7) \\
11(10)\end{array}$ & & & \\
\hline $\begin{array}{l}\text { IMC } \\
\qquad 25 \\
25-29,9 \\
\geq 30\end{array}$ & $\begin{array}{l}41(37,9) \\
43(39,8) \\
24(22,2)\end{array}$ & 26,7 & $12,0-43,5$ & 28,12 \\
\hline $\begin{array}{c}\text { Ranson } \\
\quad<3 \\
\geq 3\end{array}$ & $\begin{array}{l}81(91) \\
8(8,9)\end{array}$ & 1,1 & $0-4$ & 1 \\
\hline $\begin{array}{l}\text { APACHE } \\
\quad<7 \\
\geq 7\end{array}$ & $\begin{array}{l}54(56,8) \\
41(43,1)\end{array}$ & 6 & $0-33$ & 2 \\
\hline $\begin{array}{l}\text { Marshall } \\
\quad<3 \\
\geq 3\end{array}$ & $\begin{array}{l}53(79,1) \\
14(20,9)\end{array}$ & 0,8 & $0-5$ & 0 \\
\hline $\begin{array}{l}\text { Balthazar } \\
\text { A } \\
\text { B } \\
\text { C } \\
\text { D } \\
\text { E }\end{array}$ & $\begin{array}{c}9(8,3) \\
19(17,4) \\
9(8,3) \\
2(1,8) \\
3(2,7)\end{array}$ & & & \\
\hline $\begin{array}{l}\text { SDOM } \\
\text { Sí } \\
\text { No }\end{array}$ & $\begin{array}{c}9(8,2) \\
100(91,8)\end{array}$ & & & \\
\hline $\begin{array}{l}\text { Clasificación Atlant } \\
2012 \\
\text { Leve } \\
\text { Moderada } \\
\text { Severa }\end{array}$ & $\begin{array}{c}73(66,9) \\
17(15,6) \\
19(17,43)\end{array}$ & & & \\
\hline $\begin{array}{l}\text { Necrosis } \\
\text { Sí } \\
\text { No }\end{array}$ & $\begin{array}{c}8(7,3) \\
101(92,7)\end{array}$ & & & \\
\hline $\begin{array}{l}\text { Estado al egreso } \\
\text { Vivo } \\
\text { Muerto }\end{array}$ & $\begin{array}{c}100(91,7) \\
9(8,3)\end{array}$ & & & \\
\hline $\begin{array}{l}\text { Días de } \\
\text { hospitalización }\end{array}$ & & 15,37 & $1-74$ & 15 \\
\hline Días en UCl & & 9,34 & $1-28$ & 4 \\
\hline
\end{tabular}

APACHE: Acute Physiology and Chronic Health Evaluation; DM2: diabetes mellitus tipo 2; HTA: hipertensión arterial; SDOM: síndrome de disfunción orgánica múltiple; UCI: unidad de cuidados intensivos. 
restantes 1 presentó como causa el consumo de alcohol y 1 hipertrigliceridemia; en los 10 pacientes restantes no se encontraron datos (Tabla 2).

Tabla 2. Etiología de la pancreatitis

\begin{tabular}{lcc}
\hline \multicolumn{1}{c}{ Etiología } & $\mathbf{N .}^{\circ}$ & $\%$ \\
\hline No clara & 2 & 2,04 \\
Biliar & 91 & 92,86 \\
Alcohol & 1 & 1,02 \\
Post-CPRE & 3 & 3,06 \\
HTAG & 1 & 1,02 \\
Sin dato & 10 & 9,17 \\
\hline
\end{tabular}

*Posterior a realización de CPRE. HTAG: hipertrigliceridemia.

Se realizó tomografía axial computarizada (TAC) en 42 pacientes de los incluidos en el estudio, de los cuales se reportaron en la historia clínica como Balthazar A a 9 pacientes $(8,26 \%), \mathrm{B}$ a 19 pacientes $(17,4 \%), \mathrm{C}$ a 9 pacientes $(8,26 \%), \mathrm{D}$ a 2 pacientes $(1,83 \%)$ y E a 3 pacientes $(2,75 \%) ; 67$ pacientes $(61 \%)$ no tenían registro de esta clasificación. De los 19 pacientes con pancreatitis severa, 13 no contaron con estudio imagenológico (Tabla 3).

Tabla 3. Clasificación tomográfica por gravedad según la escala de Balthazar

\begin{tabular}{|c|c|c|c|c|c|c|}
\hline \multirow{2}{*}{ 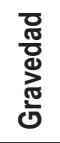 } & \multicolumn{6}{|c|}{ Escala de Balthazar } \\
\hline & A & B & C & D & $E$ & SIN TAC \\
\hline$\stackrel{0}{\Xi}$ & $\begin{array}{c}7 \\
(9,5 \%)\end{array}$ & $\begin{array}{c}16 \\
(21,9 \%)\end{array}$ & $\begin{array}{c}4 \\
(5,48 \%)\end{array}$ & $\begin{array}{c}1 \\
(1,37 \%)\end{array}$ & 0 & $\begin{array}{c}45 \\
(61,6 \%)\end{array}$ \\
\hline $\begin{array}{l}\frac{\pi}{0} \\
\frac{\pi}{\pi} \\
\frac{\pi}{0} \\
\frac{0}{2}\end{array}$ & $\begin{array}{c}1 \\
(5,8 \%)\end{array}$ & $\begin{array}{c}1 \\
(5,8 \%)\end{array}$ & $\begin{array}{c}4 \\
(23,5 \%)\end{array}$ & 0 & $\begin{array}{c}2 \\
(11,7 \%)\end{array}$ & $\begin{array}{c}9 \\
(52,9 \%)\end{array}$ \\
\hline $\begin{array}{l}\frac{\pi}{\omega} \\
\stackrel{\infty}{\infty} \\
\infty\end{array}$ & $\begin{array}{c}1 \\
(5,2 \%)\end{array}$ & $\begin{array}{c}2 \\
(10,5 \%)\end{array}$ & $\begin{array}{c}1 \\
(5,2 \%)\end{array}$ & $\begin{array}{c}1 \\
(5,2 \%)\end{array}$ & $\begin{array}{c}1 \\
(5,2 \%)\end{array}$ & $\begin{array}{c}13 \\
(68,4 \%)\end{array}$ \\
\hline
\end{tabular}

El promedio de estancia hospitalaria fue de 15,23 días e ingresaron a la UCI 35 pacientes, con un promedio de estancia de 9,34.

Dentro de los paraclínicos analizados en este estudio se encontró reportada proteína $C$ reactiva (PCR) en 75 pacientes, negativa en 29 pacientes $(26,6 \%)$ y positiva en 46 pacientes $(42,2 \%)$. De los pacientes con pancreatitis severa solo 8 (42\%) tuvieron PCR positiva (Tabla 4). En 11 pacientes se realizó procalcitonina encontrándose positiva en todos los pacientes; de estos, 4 cursaban con pancreatitis grave (Tabla 5).
Tabla 4. Valores de PCR por gravedad (Atlanta, 2012)

\begin{tabular}{lccc}
\hline \multirow{2}{*}{ Gravedad } & \multicolumn{3}{c}{ PCR } \\
\cline { 2 - 4 } & Negativa & Positiva & Sin PCR \\
\hline Leve & $20(27,4 \%)$ & $28(38,3 \%)$ & $25(34,2 \%)$ \\
Moderada & $4(23,5 \%)$ & $10(58,8 \%)$ & $3(17,6 \%)$ \\
Severa & $5(26,3 \%)$ & $8(42,1 \%)$ & $6(31,5 \%)$ \\
\hline
\end{tabular}

Tabla 5. Valores de procalcitonina según el grado de severidad

\begin{tabular}{lccc}
\hline \multirow{2}{*}{ Gravedad } & \multicolumn{3}{c}{ Procalcitonina } \\
\cline { 2 - 4 } & Sin procalcitonina & Positiva & Negativa \\
\hline Leve & $68(93,1 \%)$ & $5(6,8 \%)$ & 0 \\
Moderada & $15(88,2 \%)$ & $2(11,7 \%)$ & 0 \\
Severa & $15(78,9 \%)$ & $4(21,0 \%)$ & 0 \\
\hline
\end{tabular}

Se empleó antibiótico en 59 pacientes, de los cuales 5 tuvieron alguna clase de aislamiento bacteriano como indicación; a los otros 54 pacientes se les inició antibiótico por indicación clínica; se empleó ampicilina sulbactam en 35 pacientes $(32,1 \%)$, carbapenémico en 14 pacientes $(12,8 \%)$ y piperacilina tazobactam en 10 pacientes $(9,17 \%)$, con un promedio de 9,87 días de uso de antibiótico.

El promedio de días sin vía oral fue de 3,65; se inició vía oral en 85 pacientes $(77,98 \%)$, recibieron nutrición enteral 4 pacientes $(3,66 \%)$, nutrición parenteral 16 pacientes $(14,6 \%)$ y nutrición mixta 4 pacientes $(3,66 \%)$ (Tablas 6 y 7 ).

Tabla 6. Soporte nutricional recibido de acuerdo con la clasificación de gravedad de Atlanta, 2012

\begin{tabular}{lcccc}
\hline \multirow{2}{*}{ Gravedad } & \multicolumn{4}{c}{ Vía de nutrición } \\
\cline { 2 - 5 } & Oral & Enteral & NPT & Mixta \\
\hline Leve & $63(87,5 \%)$ & $1(1,3 \%)$ & $6(8,3 \%)$ & $2(2,7 \%)$ \\
Moderada & $12(70,5 \%)$ & 0 & $5(29,4 \%)$ & 0 \\
Severa & $10(52,6 \%)$ & $3(10,5 \%)$ & $5(26,3 \%)$ & $2(10,5 \%)$ \\
Total & 85 & 4 & 16 & 4 \\
\hline
\end{tabular}

NPT: nutrición parenteral total.

Tabla 7. Correlación entre el tipo de nutrición y el estado al egreso de los pacientes

\begin{tabular}{lccc}
\hline \multicolumn{1}{c}{ Tipo de } & \multicolumn{3}{c}{ Estado al egreso } \\
\cline { 2 - 4 } \multicolumn{1}{c}{ nutrición } & Muerto & Vivo & Total \\
\hline Oral & $4(4,7 \%)$ & $81(95,3 \%)$ & 85 \\
Enteral & 0 & $3(100 \%)$ & 3 \\
NPT & $4(25 \%)$ & $12(75 \%)$ & 16 \\
Mixta & $1(25 \%)$ & $3(75 \%)$ & 4 \\
\hline
\end{tabular}


Se realizó un estudio de vía biliar en 82 pacientes $(75,22 \%)$, por medio de ultrasonografía endoscópica (USE) en 37 pacientes (33,94\%), colangiorresonancia (CRM) en 31 pacientes $(28,44 \%)$ y CPRE en 31 pacientes $(28,44 \%)$ (Tabla 8). Se diagnosticó coledocolitiasis en 24 pacientes $(22,01 \%)$ y litiasis pancreática en 2 pacientes (1,83\%). De los 109 pacientes del estudio se encontró colelitiasis en 81 pacientes $(74,31 \%)$ y barro biliar en 16 pacientes (14,67\%). Se realizó colecistectomía en 55 pacientes $(50,45 \%)$, se realizaron 44 por laparoscopia y 9 abiertas; 11 pacientes tenían antecedente de colecistectomía.

Tabla 8. Estudio de la vía biliar

\begin{tabular}{lcc}
\hline \multicolumn{1}{r}{ Estudios } & $\mathbf{N .}^{\circ}$ & $\%$ \\
\hline CRM & 31 & 28,4 \\
USE & 37 & 34,0 \\
CPRE & 31 & 28,4 \\
\hline
\end{tabular}

Al correlacionar la edad con la severidad de la pancreatitis, se encontró que el $68 \%$ de los pacientes con pancreatitis grave era mayor de 60 años y ningún paciente menor de 25 años cursó con pancreatitis severa. La prueba de chi cuadrado $\left(\chi^{2}\right)$ mostró una significancia estadística para la edad como factor de riesgo para pancreatitis severa $(\mathrm{p}=$ 0,04) (Tabla 9).

Tabla 9. Correlación de la edad de los pacientes con la clasificación de gravedad según Atlanta, 2012

\begin{tabular}{lccc}
\hline \multicolumn{1}{c}{ Edad } & \multicolumn{3}{c}{ Gravedad } \\
\cline { 2 - 4 } & Leve & Moderada & Severa \\
\hline$<25$ & $7(9,59 \%)$ & $1(5,88 \%)$ & 0 \\
$25-59$ & $40(54,79 \%)$ & $12(70,59 \%)$ & $6(31,58 \%)$ \\
$\geq 60$ & $26(35,62 \%)$ & $4(23,53 \%)$ & $13(68,42 \%)$ \\
\hline
\end{tabular}

El IMC no mostró significancia estadística como factor de riesgo para la severidad de la pancreatitis $(p=0,8)$ (Tabla 10).

Tabla 10. Correlación del IMC con la clasificación de gravedad según Atlanta, 2012

\begin{tabular}{lccc}
\hline \multirow{2}{*}{ IMC } & \multicolumn{3}{c}{ Gravedad } \\
\cline { 2 - 4 } & Leve & Moderada & Severa \\
\hline$<25$ & $29(40,28 \%)$ & $6(35,29 \%)$ & $6(31,58 \%)$ \\
$25-29,9$ & $26(36,11 \%)$ & $9(52,94 \%)$ & $8(42,11 \%)$ \\
$>30$ & $17(23,61 \%)$ & $2(11,76 \%)$ & $5(26,32 \%)$ \\
\hline
\end{tabular}

De la totalidad de pacientes, en 8 se documentó necrosis pancreática, de los cuales ninguno tenía reportada comor- bilidad previa (Ej.: HTA, DM, cardiopatía, alcohol, entre otras). La mayoría de estos pacientes cursó con pancreatitis severa a su ingreso $(87 \%)$ y 1 paciente con pancreatitis moderada. La distribución por género mostró que la mayoría de los pacientes con necrosis era de sexo masculino ( 7 casos). La mayoría de estos pacientes se encontraba en el rango de edad de 25 a 59 años ( 5 pacientes), solo 3 pacientes mayores de 60 años y ninguno menor de 25 años. El IMC encontrado con mayor frecuencia en estos pacientes indicaba sobrepeso (50\%). La escala de severidad de Marshall solo se pudo calcular en 5 pacientes por falta de datos, de los cuales 3 dieron valores $<3$. Según la clasificación de Atlanta 2012, de los 8 pacientes que cursaron con necrosis pancreática a su ingreso se clasificaron 7 pacientes como pancreatitis severa y 1 con pancreatitis moderada (Tabla 11).

Tabla 11. Distribución de necrosis por clasificación de gravedad según Atlanta, 2012

\begin{tabular}{lcc}
\hline \multicolumn{1}{c}{ Gravedad } & $\mathbf{N}^{\circ}$ & $\%$ \\
\hline Leve & 0 & 0,00 \\
Moderada & 1 & 12,50 \\
Severa & 7 & 87,50 \\
\hline
\end{tabular}

De los 8 pacientes que cursaron con necrosis pancreática, 5 se manejaron quirúrgicamente, con un abordaje por laparotomía en 3 pacientes y 2 por laparoscopia, todos con un promedio de necrosectomía del 32,5\%. De los 3 pacientes restantes, a 1 se le realizó manejo mínimamente invasivo por medio de drenajes percutáneos y a 2 , manejo conservador (Figura 2). De los pacientes llevados a cirugía, se les practicó colecistectomía a 2 en el mismo tiempo quirúrgico.

Recibieron nutrición enteral 1 paciente $(12,5 \%)$, nutrición parenteral 3 pacientes $(37,5 \%)$, nutrición mixta 2 pacientes $(25 \%)$ y vía oral 2 pacientes (25\%) (Tabla 12).

Tabla 12. Correlación del tipo de nutrición de los pacientes de acuerdo con la presencia de necrosis

\begin{tabular}{lccccc}
\hline \multirow{2}{*}{ Necrosis } & \multicolumn{5}{c}{ Tipo de nutrición } \\
\cline { 2 - 6 } & Oral & Enteral & NPT & Mixta & Total \\
\hline No & $84(83 \%)$ & $2(2 \%)$ & $13(13 \%)$ & $2(2 \%)$ & 101 \\
Sí & $2(25 \%)$ & $1(12,5 \%)$ & $3(37,5 \%)$ & $2(25 \%)$ & 8 \\
\hline
\end{tabular}

De la totalidad de los pacientes con necrosis se reportó mortalidad en 3 pacientes (37,5\%), siendo todos manejados quirúrgicamente, 2 por vía laparoscópica y 1 por vía abierta. 2 de los 3 pacientes ( $12 \%$ ) que fallecieron tenían escala de severidad de Marshall $>4$ al ingreso y un promedio de APACHE II de 16 (Tabla 13). 


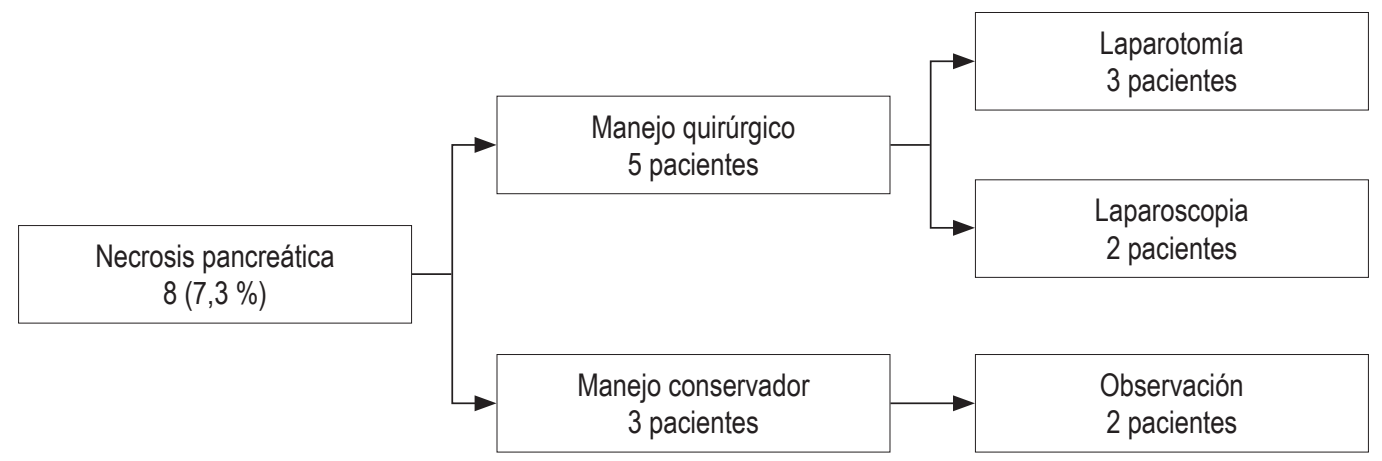

Figura 2. Manejo de la necrosis pancreática.

Tabla 13. Correlación del estado al egreso de acuerdo con la presencia de necrosis

\begin{tabular}{lccc}
\hline \multirow{2}{*}{ Necrosis } & \multicolumn{3}{c}{ Estado al egreso } \\
\cline { 2 - 4 } & Muerto & Vivo & Total \\
\hline No & $6(5,9 \%)$ & $95(94 \%)$ & 101 \\
Sí & $3(37,5 \%)$ & $5(62,5 \%)$ & 8 \\
\hline
\end{tabular}

De los 109 pacientes incluidos en el estudio, 100 pacientes $(91,74 \%)$ resolvieron su cuadro de pancreatitis y egresaron vivos de la institución, para una mortalidad reportada en 9 pacientes $(8,25 \%)$, quienes en su totalidad cursaron con pancreatitis aguda severa (Tablas 14 y 15 ).

Tabla 14. Mortalidad por clasificación de gravedad según Atlanta, 2012

\begin{tabular}{lcc}
\hline \multicolumn{1}{c}{ Gravedad } & \multicolumn{2}{c}{ Estado al egreso } \\
\cline { 2 - 3 } & Muerto & Vivo \\
\hline Leve & 0 & $73(100 \%)$ \\
Moderada & 0 & $17(100 \%)$ \\
Severa & $9(47,4 \%)$ & $10(52,6 \%)$ \\
\hline
\end{tabular}

Tabla 15. Mortalidad global

\begin{tabular}{lcc}
\hline \multicolumn{1}{c}{ Estado al egreso } & $\mathbf{N}^{\circ}$ & $\%$ \\
\hline Muerto & 9 & $8,26 \%$ \\
Vivo & 100 & $91,74 \%$ \\
\hline
\end{tabular}

\section{DISCUSIÓN}

En el análisis realizado en los pacientes incluidos en el estudio presentado se encontró que la edad $>60$ años tiene significancia estadística $(\mathrm{p}=0,04)$ como factor de riesgo para severidad. Bechien U. Wu y colaboradores, en su publicación realizada en la revista Gastroenterology en 2013, describieron como factor de riesgo para mortalidad en pancreatitis grave la edad, sin encontrar evidencia estadísticamente significativa de esta relación (5).

En el estudio realizado no se encontró relación con significancia estadística que estableciera la obesidad como un factor de riesgo para el aumento de la severidad de la pancreatitis $(p=0,8)$, a pesar de lo descrito en la literatura mundial. En una revisión sistemática publicada en octubre de 2014 por Rakesh Premkumar y colaboradores, sobre la relevancia clínica de la obesidad en la pancreatitis aguda, se encontraron 24 estudios con nivel de evidencia 1 A que indica que la obesidad está asociada con un incremento en el riesgo de pancreatitis aguda grave y solo 2 estudios reportaron que esta es un factor pronóstico independiente (6).

Las guías japonesas del manejo de la pancreatitis aguda publicadas en 2015 recomiendan la tomografía contrastada para identificar y diagnosticar complicaciones como la presencia de pancreatitis necrotizante y la extensión de los cambios inflamatorios, lo cual ayuda a definir el manejo oportuno de estos pacientes, y mejora el pronóstico del curso de la enfermedad (7). En el estudio que se documentó se encontraron 19 pacientes que cursaron con pancreatitis aguda grave, de los cuales se les realizó TAC solo a 6 pacientes $(31,6 \%)$ y, de estos, 4 cursaban con necrosis pancreática sin un adecuado reporte del porcentaje de necrosis en las historias clínicas; de los otros 4 pacientes con necrosis pancreática, en 3 el diagnóstico se realizó intraoperatorio y 1 por CMR; además, se infiere que los pacientes a quienes no se les realizó TAC fue debido a la imposibilidad del traslado del paciente por compromiso severo de su condición clínica.

En cuanto a la profilaxis antibiótica, la evidencia es controversial, aunque existen múltiples ensayos clínicos controlados que comparan antibióticos de amplio espectro frente a placebo, los cuales no han demostrado diferencias estadísticamente significativas en cuanto a la incidencia de complicaciones infecciosas, disfunción orgánica y mortali- 
dad; se ha mostrado disminución en la incidencia de estas complicaciones en los pacientes que han recibido este tipo de profilaxis, sin lograr estandarizarla incluso en pacientes con pancreatitis severa $(4,8)$.

En relación con el inicio de antibioticoterapia de amplio espectro de carácter terapéutico, múltiples estudios y guías de práctica clínica recomiendan los carbapenémicos como la mejor opción por su alta penetrancia en el tejido pancreático y su influencia en la reducción de la mortalidad; además, consideran tener en cuenta indicaciones claras para la instauración en este tipo de pacientes, entre las que se mencionan necrosis pancreática $>30 \%-40 \%$ por TAC, aislamiento microbiológico de colección peripancreática y mala respuesta clínica en pacientes con pancreatitis severa. En el presente estudio, la incidencia de pancreatitis severa fue del $17 \%$; sin embargo, el $63 \%$ de los pacientes recibió tratamiento antibiótico (principalmente ampicilina sulbactam), seguido por carbapenémicos; esto puede explicarse por la principal causa de los diferentes casos, dado por una etiología biliar, ya que en nuestro medio es muy frecuente la presentación de colecistitis aguda grados II y III asociadas con episodios de pancreatitis que, según el protocolo institucional, ameritan el inicio de cubrimiento antibiótico (8).

La pancreatitis grave asociada con complicaciones como la necrosis pancreática es relativamente frecuente dentro del curso natural de la enfermedad. En el informe realizado se encontró que 8 pacientes cursaron con dicha complicación, de los cuales 5 pacientes $(4,6 \%)$, debido a mala respuesta al tratamiento médico instaurado, inestabilidad hemodinámica y en 2 casos confirmación de hemoperitoneo, fueron llevados a manejo quirúrgico y 3 llevados a manejo médico por indicación del cirujano tratante; siguiendo esta secuencia, la mortalidad mayor se presentó en el grupo tratado quirúrgicamente con un $37,5 \%$ (3 pacientes), resultados que respaldan lo descrito en múltiples series de casos internacionales, en los que se establecen mejores resultados con el manejo conservador, como lo descrito en el metaanálisis publicado por el doctor Venigalla Pratap y colaboradores, en el que reportaron una tasa de éxito del $64 \%$ para el manejo conservador de la necrosis pancreática que fue estadísticamente significativa (riesgo relativo [RR]: 0,64; intervalo de confianza [IC] $95 \%: 0,51-0,68)$ con una tasa de mortalidad del $12 \%$ sin significancia estadística (9).

\section{CONCLUSIONES}

El estudio presentado es de tipo descriptivo, establece las características demográficas y el curso de la enfermedad de los pacientes con pancreatitis aguda incluidos las complicaciones y el manejo de estas. Con este estudio no se pretende estandarizar causalidad ni manejo de la patología descrita, solo se presenta la experiencia realizada en un hospital de cuarto nivel para contribuir con el desarrollo de estudios aleatorizados que permitan establecer guías de manejo y, de este modo, mejorar el pronóstico de los pacientes que cursen con pancreatitis aguda.

\section{REFERENCIAS}

1. Lankisch PG, Apte M, Banks PA. Acute pancreatitis. Lancet. 2016;386(9988):85-96. doi: 10.1016/S01406736(14)60649-8.

2. Yadav D, Lowenfels AB. The epidemiology of pancreatitis and pancreatic cancer. Gastroenterology. 2013;144(6):1252-61. doi: $10.1053 /$ j.gastro.2013.01.068.

3. Haney JC, Pappas TN. Necrotizing pancreatitis: diagnosis and management. Surg Clin North Am. 2007;87(6):143146. doi: 10.1016/j.suc.2007.08.013.

4. LovedayBPT, SrinivasaS, VatherR, MittalA, PetrovMS, Phillips ARJ, et al. High quantity and variable quality of guidelines for acute pancreatitis: a systematic review. Am J Gastroenterol. 2010;105(7):1466-76. doi: 10.1038/ajg.2010.137.

5. Wu BU, Johannes RS, Sun X, Tabak Y, Conwell DL, Banks PA. The early prediction of mortality in acute pancreatitis: a large population-based study. Gut. 2008;57(12):1698-703. doi: 10.1136/gut.2008.152702.

6. Premkumar R, Phillips ARJ, Petrov MS, Windsor JA. The clinical relevance of obesity in acute pancreatitis: Targeted systematic reviews. Pancreatology. 2016;15(1):25-33. doi: 10.1016/j.pan.2014.10.007.

7. Yokoe M, Takada T, Mayumi T, Yoshida M, Isaji S, Wada $\mathrm{K}$, et al. Japanese guidelines for the management of acute pancreatitis: Japanese Guidelines 2015. J Hepatobiliary Pancreat Sci. 2015;22(6):405-32. doi: 10.1002/jhbp. 259.

8. Serrablo A, Tejedor L, Martínez J. Antibiotics in severe acute pancreatitis. Cent Eur J Med. 2014;9(4):565-73. doi: 10.2478/s11536-013-0281-3.

9. Mouli VP, Sreenivas V, Garg PK. Efficacy of conservative treatment, without necrosectomy, for infected pancreatic necrosis: a systematic review and meta-analysis. Gastroenterology. 2013;144(2):333-340.e2. doi: $10.1053 /$ j.gastro.2012.10.004. 